\title{
Establishing Clinical Skills Center in a Developing Country-LMDC Experience
}

\author{
Ibnerasa, S.N. ${ }^{1}$, Daud, S. $^{2}$, Chaudrhy, A.M. ${ }^{3}$
}

\begin{abstract}
Clinical skills centers have become an integral part of the curricular requirements in most modern medical schools and postgraduate centers; worldwide. There has also been a growing interest in the development of clinical skills laboratories in the developing countries in the past decade. Lahore Medical and Dental College, Lahore, Pakistan took the initiative of developing a low cost clinical skills lab in July 2010.The development of skills lab required a multi-step approach. A six steps approach of Syme-Drant (2005) was modified and used to develop clinical skills lab teaching and learning protocols. The objective of this study is to describe the stages involved in the development of a low cost, clinical skills training center in a medical college in a developing country.
\end{abstract}

Key Words: Education, medical, undergraduate, clinical skills, teaching material

\section{Introduction}

Procedural competence requires a range of training initiatives, from bedside teaching to that of simulation in a skills-lab (Connick et al., 2009).Clinical skills laboratories have become an integral part of the curricular requirements in most modern medical schools and postgraduate centers worldwide and developing countries are no exception (Stark \& Fortune 2003).Lahore Medical and Dental College (LMDC), Lahore, Pakistan developed Clinical skills laboratory in 2010.The main purpose behind development was improvement in students' clinical skills as clinical teaching at LMDC was hospital-based, and on patient bed-side only.

${ }^{1}$ Professor, Director Skills Lab and Professor of Histopathology

${ }^{2}$ Professor, Co-Director, Department of Medical Education \& Chairperson Medical Education Committee,

Head, Department of Community Medicine

${ }^{3}$ Professor, Principal and Head Department of Surgery and Director Department of Medical Education

Lahore Medical and Dental College, Lahore, Pakistan

Corresponding Author:

Dr. Shazia Nilofar Ibnerasa, Professor of Histopathology and Director Skills Laboratory, Lahore Medical and Dental College, Lahore 54600, Pakistan.

E-mail: Shazia.nilofar@Imdc.edu.pk
Students' participation in clinical skills teaching was voluntary with limited ward teaching time. It is not possible for every student to practice clinical skills individually.

Keeping in view the evolutionary steps that were taken at LMDC, the objective of this article is to discuss the stages involved in the development of a low cost, clinical skills lab in a developing country. The aim is to describe a step wise approach which can be easily followed by other medical colleges, who have limited resources and wish to develop a similar laboratory in their institutes.

Development of a clinical skills laboratory requires work in three different areas. Selection of a designated area where clinical skills teaching activities could be organized, development of skills teaching curriculum and procurement/development of teaching material.

\section{Space requirement and allocation.}

A 2000 square feet area was identified and selected. It was equipped with furniture for small group teaching and with complete audiovisual (multi-media) system. 


\section{Development of list of skills and formation of teaching plan (core- curriculum).}

Voluntary participation in clinical skills teaching was requested and a clinical skill curriculum working group was formed. This group included members of the Department of
Medical Education, all Clinical Department Professors and their nominated teachers (who volunteered for skills teaching). A six steps approach was developed following the steps of principal curriculum development protocols (stages) of Syme-Drant (2005) cited by Hassan (2007) to develop clinical skills laboratory teaching protocols (Figure 1).

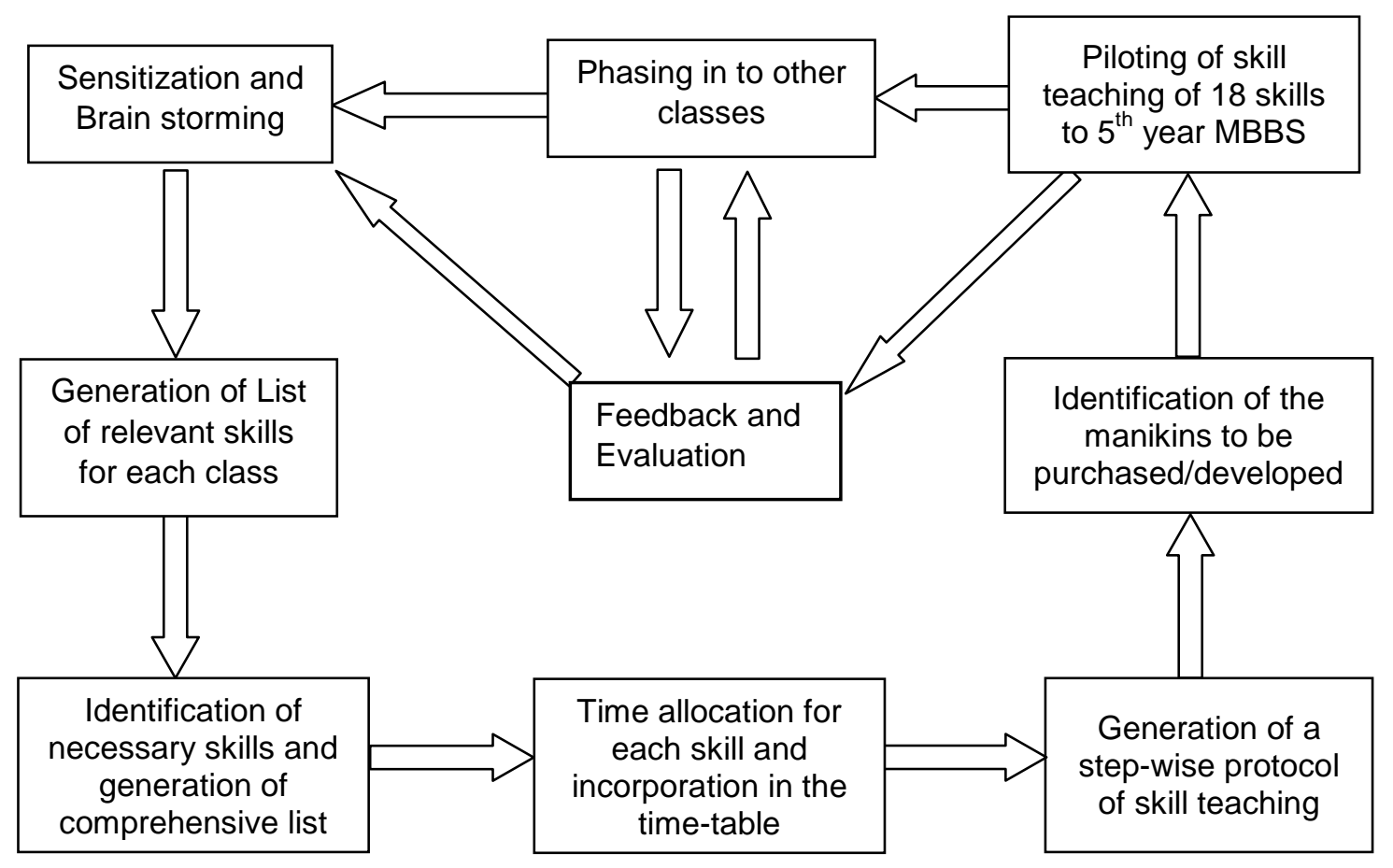

Figure 1: A modified version of steps of principal curriculum development protocols (stages) of Syme-Drant (2005) cited by Hassan (2007)

These steps were as follows:

\section{Step-I: Faculty Sensitization and Brain Storming}

All the basic sciences and clinical faculty professors and their nominated teachers were requested to update their respective teaching faculty about this new venture and to identify teachers who would like to volunteer and accept the challenge of teaching clinical skills to the undergraduate students. They were also asked to prepare a list of clinical skills which in their opinion, a fresh medical graduate should be knowing.

\section{Step-II: Selection and generation of Skills list}

The topic was open for debate till a consensus was reached in defining a list of clinical skills for each class i.e. from first year to final year MBBS. Table 1 shows the list of essential skills as approved by the all the faculty members. A step-wise performance protocol was developed for each respective skill.

\section{Steps-III: Curriculum Integration}

Easier skills were kept in the initial (preclinical) years, while complex skills kept in the final clinical years. Integration of the skills with the taught curriculum was done wherever possible and clinical skills teaching were aligned with the students' clinical rotations timetable.

Step-IV: Making a Timetable, deciding the Teaching Methodology and monitoring of student attendance

It was decided that skills lab teaching time will be taken out from students' practical time for pre-clinical years and their ward time for clinical years. Adopting small group learning 
strategy, skills teaching was planned once every week, for two hours for a group of 8-10 students.

\section{Step-V: Piloting and Feedback}

Piloting of clinical skills teaching was done on final year students. The perception of students and teaching faculty were then taken on a five point Likert scale (Ibnerasa, 2011). Teaching programme was modified according to the feedback received by the students and the faculty.

\section{Step-VI: Annual review}

It was decided that after the completion of one year of skills teaching, the teaching curriculum will be analysed for its strengths and weaknesses.

\section{Table 1: List of Essential Clinical Skills Taught in Five Years}

\begin{tabular}{ll}
\hline FIRST YEAR SKILLS \\
\hline 1 & Introduction to bandages and dressings \\
2 & How to measure different pulses \\
3 & How to take blood pressure \\
4 & How to approach a patient for medical \\
5 & What are Aseptic techniques \\
\hline SECOND YEAR SKILLS \\
\hline 6 & How to lift and transfer an injured patient \\
7 & How to give an Intramuscular Injection \\
8 & How to check blood sugar from a glucometer \\
\hline THIRD YEAR SKILLS \\
\hline 9 & How to take Blood sample for blood culture \\
10 & How to take a Throat swab \\
11 & How to apply Cardiac monitoring/leads \\
12 & How to Nebulization a patient \\
13 & Learning Hand washing/Scrubbing \\
\hline FOURTH YEAR SKILLS \\
\hline 14 & How to perform Breast examination \\
15 & How to perform a Tru-cut biopsy \\
16 & Different Skin Incisions \\
\hline 17 & Performing BLS \\
\hline
\end{tabular}

\section{Procurement of manikins, models and simulators}

Taking help from the different catalogues available in the market for clinical skills teaching material, low cost models were developed from locally available raw materials. These models were created with plastic, sponge and rubber (Figure 2).

\section{Discussion}

The structural and functional organization of a low cost, clinical skills laboratory at Lahore Medical and Dental College within a year, is a proof that clinical skills centers of reasonable standard can be developed and/or replicated
18 Removal of foreign body by Hemlich's maneuver

19 How to perform Phlebotomy \& make a blood film.

20 How to perform Fine Needle aspiration

21 How to perform Lumber puncture

22 How to take a High vaginal Swab

23 How to insert an Intra-uterine contraceptive device

24 Plaster of Paris application on fracture

25 Closed fracture splint application

\section{FINAL YEAR SKILLS}

26 How to perform End tracheal intubation

27 How to perform Nasogastric intubation

28 Ventilator monitoring

29 How to perform Newborn resuscitation

30 Steps of doing an Episiotomy

31 How to perform Forceps delivery

32 Performing Normal and breech delivery

33 ACLS

34 Primary trauma care

35 How to pass a Chest tube

36 How to perform Venous cut down

37 Performing an Ostomy

38 How to pass Foleys catheter

in a developing country such as Pakistan. The overall response of the students and teachers participating in the skills sessions was positive. The students expressed that clinical skills laboratory teaching will enhance their procedural confidence while dealing with actual patients in clinics. Setting up of a Clinical Skills Center requires some initial capital and manikin purchase costs followed by day to day expenditure and ongoing costs of personnel and equipment. The fear of financial burden can be a deterrent in establishing skills lab in developing countries. Such problems have been mentioned by another institute in Pakistan as constraining impediments (Khaja 2008). 
a- Cannula Insertion

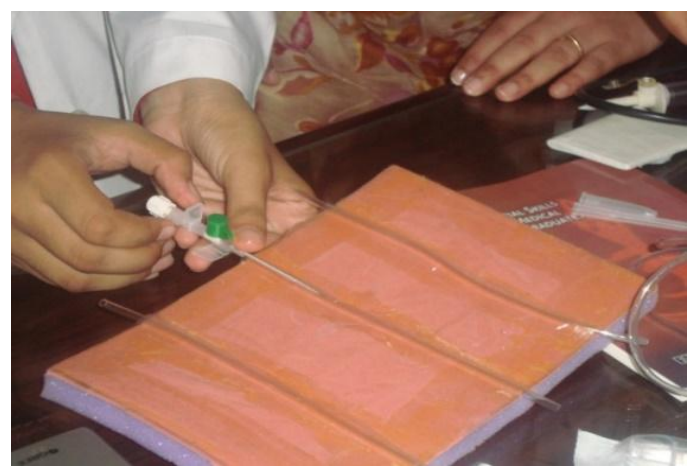

c- Episiotomy

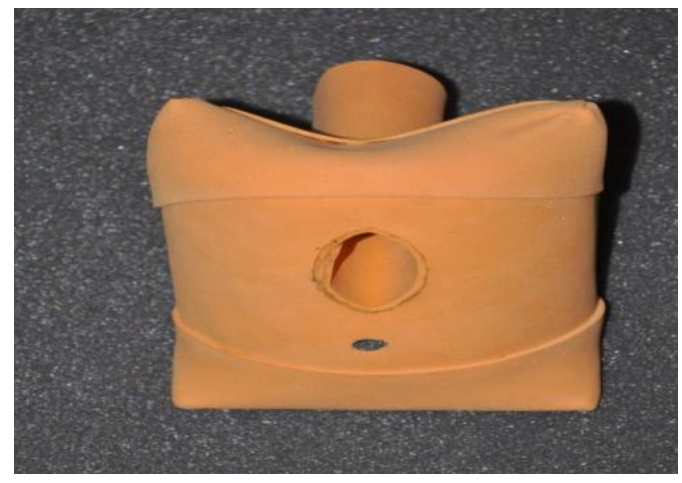

\section{b- Phlebotomy practice band for arm}

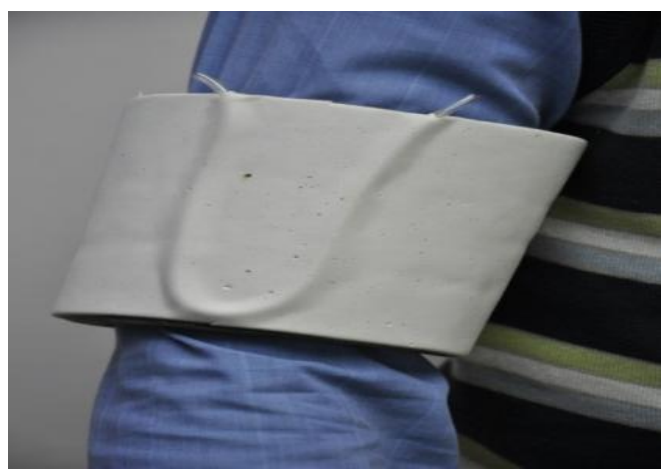

d-Tracheostomy

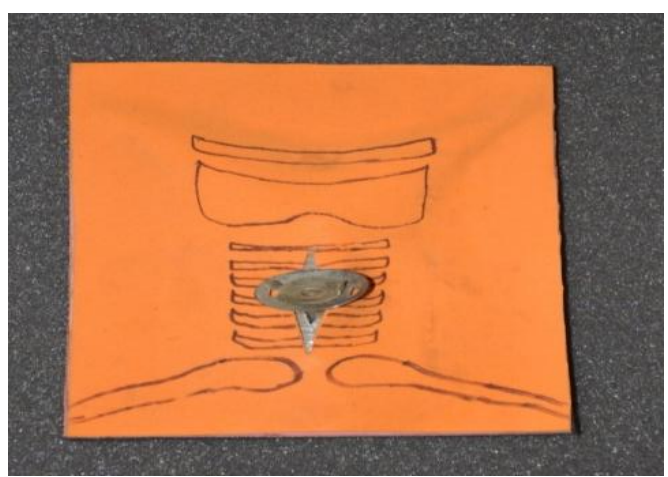

Figure 2: Different clinical skills teaching model

In LMDC, almost everything from the space to the furniture and most of the teaching material were generated from the institute. This was the main factor in controlling the amount of initial capital required to start a clinical skills center. Clinical skills lab faculty comprised of part-time clinical teachers from different specialties', who were motivated enough to volunteer for clinical skills teaching. This was another key factor in successful functioning of the center. Development of practice models from locally available material helped in cutting the running expense of teaching material. It was not only cost effective but also had the advantage of being used as disposable teaching material during sessions. It also helped in maintaining a ready supply of the teaching material for subsequent sessions. Clinical skills laboratory at LMDC is an example of a sustainable model as locally manufactured teaching material was developed in the institute.

\section{Conclusion}

Clinical skills labs offer the educational community a model of teaching, which appears to offer benefits and positive longterm impact on undergraduate medical students. However, a successful and sustainable skills training program require dedicated planning and a structured curriculum.

\section{References}

Ahmed, A.M., (2008) Role of clinical skills centres in maintaining and promoting clinical teaching, Sudan Journal of Public Health, 3, 2, pp. 94103.

Bligh, J. (1995) The clinical skills unit, Postgraduate Medical Journal, 7, 1, pp. 730-732. 
Connick, R.M., Connick, P., Klotsas, A.E., Tsagkaraki, P.A. \& Gkrania-Klotsas, E. (2009) Procedural confidence in hospital based practitioners: Implications for the training and practice of doctors at all grades, BMC Medical Education, 9, 1, pp. 2.

Hassan, S. (2007) How to develop a core curriculum in clinical skills for undergraduate medical teaching in the school of medical sciences at Universiti Sains Malaysia? The Malaysian Journal of Medical Sciences, 14, 2, pp. 4-10.

Higher Education Commission, Pakistan, viewed 3 March 2016, from http://www.hec.gov.pk /InsideHEC/Divisions/AECA/CurriculumRevisio n/Documents/MBBS-Draft-2010-11.pdf

Ibnerasa, S.N., Hashmi, N.R. \& Chaudrhy, A.M. (2011) Staff and student perceptions regarding a clinical skills laboratory learning experience, International Journal of Ocean and Climate Systems, 5, 2, pp. 126- 129.

Khaja, H., Quadri, M., Rahim, M.F., Alam, A.Y., Jaffery, T., Zaidi, Z., \& Iqbal, M. (2008) The structure and function of a new Clinical Skills and Medical Informatics Laboratory (SCIL) in a developing countre-a two year institutional experience, Juvenile Products Manufacturers Association, 58,11, pp. 612-615.

LSUHSC, viewed 28 April 2016, from http://www.medschool./suhsc.edu/medical_edu cation/undergraduate/spm/Curriculum/Core_Co mpetencies.asp.

Nasco, viewed 27 April 2016, from https://www.enasco.com/healthcare/

Shaikh, S.H. (2009) A Reform Agenda Outline for Medical Education in Pakistan, Journal of the College of Physicians and Surgeons Pakistan, 19, 6, pp. 331-332.

Stark, P. \& Fortune, F. (2003) Teaching Clinical Skills in Developing Countries: Are Clinical Skills Centres the Answer? Education for Health, 6, 3, pp. 298-306.

The University of Dundee, viewed 27 April 2016, from https://medicine.dundee.ac.uk/medicaleducation-institute/clinical-skills-centre. 